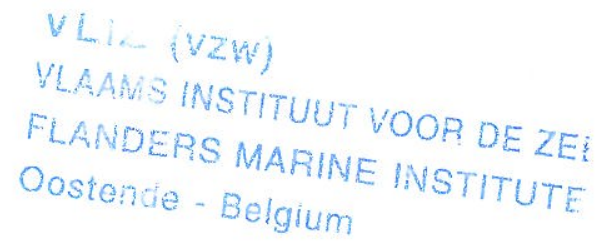

\title{
Reproductive performance, lipids and fatty acids of mud crab Scylla serrata (Forsskål) fed dietary lipid levels
}

\author{
Veronica R Alava ${ }^{1}$, Emilia T Quinitio ${ }^{1}$, Jennette B de Pedro ${ }^{1}$, Zenith G A Orosco ${ }^{1}$ \& Mathieu Wille ${ }^{2}$ \\ ${ }^{1}$ Aquaculture Department, Southeast Asian Fisheries Development Center, Buyuan, Tigbauan, Iloilo, Philippines \\ ${ }^{2}$ Laboratory of Aquaculture \& Artemia Reference Center, Ghent University, Rozier, Ghent, Belgium
}

Correspondence: V R Alava, Aquaculture Department, Southeast Asian Fisheries Development Center, Buyuan, Tigbauan 5021, Iloilo, Philippines. E-mail: vralava@aqd.seafdec.org.ph

\begin{abstract}
Natural food (NF, control), artificial diets (AD) containing total lipid levels of $10 \%, 12 \%$ and $14 \%$ (AD10 $\mathrm{AD} 12$ and $\mathrm{AD} 14)$ and their combinations (AD10+NF, $\mathrm{ADI} 2+\mathrm{NF}$ and $\mathrm{ADI}+\mathrm{NF}$ ) were fed for 112 days to pond-sourced eyestalk-ablated mud crab Scylla serrata $(625 \pm 6.4 \mathrm{~g})$ in tanks in order to determine their effects on reproduction and lipid profiles in broodstock tissues and zoeae. Crabs fed NF had the highest number of spawning followed by crabs fed $\mathrm{AD} 10+\mathrm{NF}$ and ADl4+NE. Higher offspring production (number of zoeae) was obtained from crabs fed NF and AD+NF than from AD. As dietary total lipid levels increased, total lipid of broodstock ovaries, hepatopancreas, muscle and zoeae correspondingly increased in which $\mathrm{AD}+\mathrm{NF}$ promoted higher levels than $\mathrm{AD}$. Increased dietary total lipid levels enhanced lipid classes such as triacylglycerols and phosphatidyl choline levels in zoeae, all higher in crabs fed $A D+N F$ than in AD. The major fatty acids in zoeae, particularly 16:0, 18:0, 18:1n-9 and 20:4n-6, 20:5n-3 and 22:6n-3, were higher in crabs fed $\mathrm{AD}+\mathrm{NF}$ than in $\mathrm{AD}$, the contents corresponding to broodstock dietary total lipid levels. A $10 \%$ total lipid in AD in combination with NF was sufficient to provide the essential lipids in crabs in the improvement of larval production and quality.
\end{abstract}

Keywords: mud crab, Scylla serrata, dietary lipid levels, reproduction, lipid classes and fatty acids

\section{Introduction}

Mud crab Scylla serrata (Forsskål) has been recognized as an important portunid species for commer- cial culture. It requires simple culture methods, grows to a large size and commands a high price due to the rising demand for crab meat in both domestic and international markets. Crab culture in the Philippines is an important source of income for small-scale crab farmers in coastal communities.

A major obstacle in further developing mud crab aquaculture is the inadequate supply of seed that is presently collected from the wild. Although spawning of captive crabs has been reported to occur year-round, larval survival still needs to be improved. Artificial propagation techniques for mud crab have been established at the Southeast Asian Fisheries Development Center Aquaculture Department (SEAFDEC AQD) (Quinitio \& Parado-Estepa 2003) but information on the nutritional factors that influence ovarian maturation, spawning and larval quality on crabs is limited. Hence, studies on the nutritional requirements of crab broodstock aimed to improve reproductive performance and larval quality are necessary.

Lipids and fatty acid composition of crustacean broodstock diets have been identified as major dietary factors that determine successful reproduction and egg quality (Middleditch, Missler, Ward, McVey, Brown \& Lawrence 1979; Middleditch, Missler, Hines, McVey, Brown, Ward \& Lawrence 1980; Cahu, Fauvel \& Aquacop 1986; Millamena 1989; Bray, Lawrence \& Lester 1990; Cahu, Guillaume, Stephan \& Chim 1994). Lipids are one of the main sources of metabolic energy and have important functions as cytoplasm and membrane constituents of cells affecting structural and physiological properties. Wild and pondsourced crab $S$. serrata during ovarian maturation and spawning indicated that the ovaries and newly 
spawned eggs contain high levels of highly unsaturated fatty acids (HUFA) such as arachidonic acid (ArA, 20:4n-6), eicosapentaenoic acid (EPA, 20:5n-3) and docosahexaenoic acid (DHA, 22:6n-3) (Alava, Quinitio, de Pedro, Orosco, Priolo \& Wille 2007). Moreover, phospholipids particularly phosphatidyl choline $(\mathrm{PC})$ were also accumulated in large amounts in ovaries and newly spawned eggs. Hence, artificial diets $(\mathrm{AD})$ were formulated to incorporate these essential lipids at graded levels alone or in combination with natural food (NF) and fed to adult $S$. serrata to determine their effects on the reproductive performance and quality of broodstock tissues and newly hatched zoeae in terms of lipid class and fatty acid contents.

\section{Materials and methods}

\section{Experimental diets}

Dietary treatments consisted of NF (control), AD containing $10 \%, 12 \%$ and $14 \%$ total lipids (AD10, AD12, $\mathrm{AD} 14)$ and combination of $\mathrm{AD}$ and $\mathrm{NF}$ (AD10+NF, $\mathrm{AD} 12+\mathrm{NF}, \mathrm{AD} 14+\mathrm{NF})$. In AD, the dietary lipids used were squid oil and soybean lecithin added at a 2:1 ratio (Table 1). The finely ground feed ingredients were mixed thoroughly, steam-conditioned during extrusion into 10-mm-diameter pellets, air dried and stored in a cold room until use. Mussel meat (Perna viridis), squid (Loligo sp.) and fish biomass (Leiognathus sp.) were given on a daily rotation. The dietary treatments were fed daily for 112 days at 1.0\% (dry matter basis) of the crab biomass per day, half in the morning and half in the afternoon. For the combination diet, NF was given in the morning and the dry pellets in the afternoon. The test diets and proximate composition, lipid class and fatty acid contents, are shown in Tables $1-3$ respectively.

\section{Animals}

Scylla serrata broodstock grown in brackishwater ponds in New Washington, Aklan (Panay Island), and fed with fish biomass and small bivalves Potamocorbula sp. were collected and transported to SEAFDEC AQD, Tigbauan, Iloilo, Philippines. The female crabs $(625 \pm 6.4 \mathrm{~g}$ body weight) in the intermolt stage with early maturing ovary (yellow colour) were disinfected, unilaterally eyestalk-ablated, tagged and stocked in the seven circular concrete $12-\mathrm{m}^{3}$ tanks. The colour of the gonad was seen by depressing the first abdominal segment below the carapace. Each tank was divided into two compartments where five crabs were stocked per compartment. Tagging, disinfection and maintenance of broodstock in maturation tanks were carried out following Millamena

Table 1 Ingredient composition and proximate analysis of artificial diets (AD) and natural food (NF) fed to mud crab Scylla serrata

\begin{tabular}{|c|c|c|c|c|c|c|}
\hline \multirow[b]{2}{*}{ Ingredients } & \multicolumn{3}{|l|}{ AD } & \multicolumn{3}{|l|}{$\mathrm{NF}^{*}$} \\
\hline & $A D 10$ & AD 12 & AD 14 & Squid & Fish & Mussel \\
\hline Squid oil & 3.50 & 5.00 & 6.50 & & & \\
\hline Soy lecithin & 1.75 & 2.50 & 3.25 & & & \\
\hline Rice bran & 9.75 & 7.50 & 5.25 & & & \\
\hline Common ingredients $\dagger$ & 85.00 & 85.00 & 85.00 & & & \\
\hline \multicolumn{7}{|c|}{ Proximate analysis (\% dry matter) } \\
\hline Crude protein & 43.05 & 42.88 & 43.38 & 78.51 & 71.92 & 66.70 \\
\hline Crude fat & 10.57 & 12.56 & 14.61 & 5.52 & 12.00 & 6.02 \\
\hline Crude fibre & 2.18 & 1.97 & 1.87 & 1.32 & 0.91 & 0.90 \\
\hline Ash & 16.60 & 16.31 & 16.06 & 8.01 & 13.32 & 15.22 \\
\hline Nitrogen-free extract & 27.60 & 26.28 & 25.08 & 6.64 & 1.85 & 11.16 \\
\hline Energy $\left(\mathrm{MJ} \mathrm{kg}^{-1}\right) \ddagger$ & 18.09 & 18.61 & 19.15 & 20.04 & 20.37 & 18.50 \\
\hline $\mathrm{P} / \mathrm{E}$ ratio (mg protein $\mathrm{kJ}^{-1}$ ) & 23.79 & 22.99 & 22.17 & 39.17 & 35.29 & 36.04 \\
\hline
\end{tabular}

*Squid Loligo sp., fish Leiognathus sp. and mussel meat Perna viridis.

†Common ingredients ( $\mathrm{g}$ per $100 \mathrm{~g}$ ): fish meal Chilean, 25.0; Acetes meal, 20.0; squid meal, 8.0; bread flour, 17.5; seaweed Gracilaria sp., 5.0; Aquatech binder, 0.5; carboxy methyl cellulose, 1.0; vitamin mix, 3.0; mineral mix, 3.0; dicalcium phosphate, 1.5; phoshitan C, 0.5 [vitamin and mineral mixes Kanazawa (1981)]. Alpha-tocopheryl acetate was added at $4 \mathrm{mg}$ per per cent squid oil per $\mathrm{kg}$ of diet. $\ddagger$ Computed as 21.3 for protein, 39.5 for lipids and $17.2 \mathrm{MJ} \mathrm{kg}^{-1}$ for carbohydrates (Cuzon \& Guillaume 1997) 
Table 2 Lipid class contents (mg g ${ }^{-1}$ dry weight) of artificial diets (AD) and natural food (NF) fed to mud crab Scylla serrata

\begin{tabular}{|c|c|c|c|c|c|c|}
\hline & \multicolumn{3}{|l|}{$A D$} & \multicolumn{3}{|l|}{ NF } \\
\hline & AD10 & AD12 & AD14 & Squid & Fish & Mussel \\
\hline Total lipids & 103.70 & 114.70 & 134.81 & 81.15 & 93.84 & 33.05 \\
\hline Total NL & 86.27 & 89.09 & 110.72 & 13.22 & 71.32 & 9.22 \\
\hline $\mathrm{CHO}$ esters & 1.67 & 0.94 & 0.97 & 0.11 & 0.24 & 0.33 \\
\hline TAG & 24.97 & 36.88 & 52.41 & 0.22 & 62.82 & 1.74 \\
\hline FFA & 35.09 & 35.68 & 26.28 & 0.91 & 6.66 & 0.10 \\
\hline $\mathrm{CHO}+\mathrm{DAG}$ & 15.52 & 15.59 & 16.17 & 12.00 & 1.73 & 7.33 \\
\hline Total PL & 17.43 & 24.12 & 25.61 & 68.31 & 20.62 & 21.83 \\
\hline$P E+P A$ & 8.19 & 13.19 & 14.83 & 20.72 & 5.64 & 6.92 \\
\hline$P S+P I$ & 0.02 & 0.65 & 0.81 & 10.63 & 2.11 & 3.05 \\
\hline PC & 0.98 & 1.09 & 1.76 & 32.64 & 12.00 & 11.22 \\
\hline $\mathrm{SPH}$ & 0.03 & 0.58 & 0.61 & 4.13 & 1.03 & 0.74 \\
\hline
\end{tabular}

CHO, cholesterol; TAG, triacylglycerols; FFA, free fatty acids; DAG, diacylglycerols; PE, phosphatidyl ethanolamine; PA, phosphatidic acid; PS, phosphatidyl serine; PI, phosphatidyl inositol; PC, phosphatidyl choline; SPH, spingomyelin; LPC, lyso-phosphatidyl choline; NL; neutral lipids; PL, polar lipids.

Table 3 Fatty acid contents (mg g ${ }^{-1}$ dry weight) of artificial diets (AD) and natural food (NF) fed to mud crab Scylla serrata

\begin{tabular}{|c|c|c|c|c|c|c|}
\hline \multirow[b]{2}{*}{ FAME } & \multicolumn{3}{|l|}{$A D$} & \multicolumn{3}{|l|}{ NF } \\
\hline & AD10 & AD12 & AD14 & Squid & Fish & Mussel \\
\hline $14: 0$ & 6.91 & 6.51 & 7.15 & 2.52 & 5.05 & 2.04 \\
\hline $16: 0$ & 22.20 & 23.41 & 26.70 & 24.91 & 29.44 & 6.42 \\
\hline $16: 1(n-7)$ & 6.68 & 6.68 & 7.59 & 0.93 & 11.73 & 4.14 \\
\hline $18: 0$ & 5.60 & 6.08 & 7.05 & 3.64 & 10.62 & 1.23 \\
\hline $18: 1(n-9)$ & 13.04 & 14.06 & 15.74 & 6.22 & 18.03 & 2.04 \\
\hline $18: 2(n-6)$ & 14.30 & 16.14 & 18.99 & 0.23 & 1.15 & 0.65 \\
\hline $18: 3(n-3)$ & 1.95 & 2.37 & 3.01 & 0.02 & 0.55 & 2.93 \\
\hline $18: 4(n-3)$ & 0.56 & 0.54 & 0.64 & 0.03 & 0.64 & 1.42 \\
\hline $20: 1(n-9)$ & 0.67 & 0.85 & 0.93 & 2.72 & 4.65 & 2.45 \\
\hline $20: 4(n-6)$ & 1.51 & 1.66 & 1.90 & 3.33 & 2.03 & 1.83 \\
\hline $20: 5(n-3)$ & 4.43 & 5.39 & 6.73 & 10.14 & 4.53 & 5.04 \\
\hline $22: 5(n-3)$ & 0.70 & 0.82 & 1.01 & 0.55 & 1.35 & 0.42 \\
\hline $22: 6(n-3)$ & 11.07 & 14.51 & 18.90 & 26.16 & 2.62 & 3.00 \\
\hline Total lipids & 103.70 & 114.70 & 134.80 & 81.56 & 93.82 & 33.05 \\
\hline Sum saturates & 38.04 & 39.44 & 44.77 & 31.55 & 46.63 & 9.64 \\
\hline Sum monoenes & 24.12 & 26.17 & 29.67 & 9.94 & 34.74 & 8.53 \\
\hline Sum n-3 FA & 18.97 & 24.06 & 30.79 & 36.65 & 9.46 & 12.64 \\
\hline Sum n-6 FA & 17.23 & 19.40 & 22.82 & 3.57 & 3.14 & 2.46 \\
\hline Sum n-3 HUFA & 16.46 & 21.15 & 27.15 & 36.64 & 8.45 & 8.34 \\
\hline Ratio $n-3 / n-6$ FA & 1.10 & 1.24 & 1.35 & 10.33 & 3.13 & 5.32 \\
\hline
\end{tabular}

FAME, fatty acid methyl esters; HUFA, highly unsaturated fatty acids.

and Quinitio (2000). The crabs fed AD alone were gradually weaned to the test diets for 10 days. Feeding, moulting, mortality and presence of females with spawned eggs attached to the pleopods (berried) were monitored daily. Berried females were transferred to 300-500-L circular fibre glass tanks for egg incubation and hatching. Newly hatched zoeae were collected and rinsed thoroughly for lipid analysis.

\section{Total lipid, lipid class and fatty acid analyses}

Natural food and artificial feeds were analysed for proximate composition according to standard methods (AOAC 1984). After 112 days, ovaries, hepatopancreas and muscle of crabs were dissected. These tissues and the newly hatched zoeae were extracted for total lipids following the method of Folch, Lees 
and Sloane-Stanley (1957). Neutral (NL) and polar lipids (PL) were separated by two solvent systems using thin layer chromatography in the Chromatographic Analyzer (Iatroscan, MK-5, Tokyo, Japan) with a hydrogen flame ionization detector (FID). The first system hexane, diethyl ether and formic acid 98\% (85:15:0.04, v/v/v) for 40 min separated the NL classes from total PL. After partial scanning of NL, the same rods with the remaining PL were developed in the solvent system chloroform-methanol-water (70:35:3.5, v/v/v) for $45 \mathrm{~min}$ to separate the PL classes. Thereafter, a full scan was performed in nine replicates per sample.

The preparation of fatty acid methyl esters from the total lipids was carried out by using acetylchloride/methanol mixture (1:20 v/v) as esterification agent (Lepage \& Roy 1984) and the fatty acid compositions were then determined by using Chrompack CP9001 gas chromatograph (Chrompack, Macclesfield, UK), equipped with a polar $50 \mathrm{~m}$ capillary column, BPX70 $(50 \mathrm{~m} \times 0.32 \mathrm{~mm}$ ID, $0.25 \mu \mathrm{m}$ layer thickness; SGE Analytical Science, Ringwood, Victoria, Australia), with hydrogen FID and using helium as carrier. Integration and calculations were carried out on a computer with a software program Maestro (Chrompack).

Lipid classes and fatty acids were identified using authentic standard reference mixtures (Nu-ChekPrep., Elysian, MN, USA), and results were presented as $\mathrm{mg} \mathrm{g}^{-1}$ dry weight (DW).

\section{Statistical analyses}

The total lipids, lipid classes and fatty acids of broodstock tissues and zoeae from different dietary treatments were compared using analysis of variance followed by Duncan's multiple range test to deter- mine significant differences among treatment means at $P<0.05$ (Gomez \& Gomez 1984).

\section{Results}

The duration from ablation to spawning (32-56 days) and embryonic development (10-14 days) did not vary significantly $(P>0.05)$ among treatments. However, crabs fed NF alone or in combination with $A D$ had significantly $(P<0.05)$ higher number of zoeae than those fed AD alone (Table 4).

The total lipid contents of crab zoeae were higher in $\mathrm{AD}+\mathrm{NF}$ than in $\mathrm{AD}$ and these increased as broodstock dietary lipid level increased (Table 5). Total lipid level of zoeae in the control was close to that of $\mathrm{AD} 10+\mathrm{NF}$. Likewise, the total lipid contents of ovaries, hepatopancreas and muscle of crabs increased with dietary lipid levels and these were higher in crabs fed $\mathrm{AD}+\mathrm{NF}$ than in $\mathrm{AD}$ alone (Tables 5 and 6). Hepatopancreatic lipids were higher than ovarian lipids while those of muscles were lowest.

Total NL and PL contents of zoeae improved with increased maternal dietary lipid levels (Table 5). Reflecting the broodstock diets, the total NL of zoeae was more than their total PL. Zoeal triacylglycerols (TAG), free fatty acids (FFA), cholesterol+diacylglycerol (CHO+DAG), $\mathrm{PC}$ and phosphatidyl ethanolamine+ phosphatidic acid $(\mathrm{PE}+\mathrm{PA})$ improved with maternal dietary lipid levels, in which $\mathrm{AD}+\mathrm{NF-fed} \mathrm{crabs} \mathrm{were}$ higher than in crabs fed AD alone. In ovaries, NL was also higher than PL (Table 5). Dietary lipid levels increased the ovarian TAG, FFA and CHO esters in crabs fed $\mathrm{AD}$ as well as $\mathrm{CHO}+\mathrm{DAG}$ in crabs fed $\mathrm{AD}+\mathrm{NE}$. The ovarian phospholipids $\mathrm{PC}, \mathrm{PE}+\mathrm{PA}$ and sphingomyelin increased corresponding to dietary levels in which crabs fed $A D+N F$ had higher levels than those fed $\mathrm{AD}$ alone. In hepatopancreas, NL was

Table 4 Reproductive performance of mud crab Scylla serrata fed artificial diets (AD) containing graded total lipid levels with or without natural food (NF)

\begin{tabular}{lllllr}
\hline Treatment & $\begin{array}{l}\text { Ablation to } \\
\text { spawning (days) }\end{array}$ & $\begin{array}{l}\text { Spawning to } \\
\text { hatching (days) }\end{array}$ & $\begin{array}{l}\text { Mean zoeae/spawn } \\
\left(\times 10^{3}\right)\end{array}$ & $\begin{array}{l}\text { Repeat } \\
\text { spawns }\end{array}$ & $\begin{array}{l}\text { Survival } \\
\text { rate }(\%)\end{array}$ \\
\hline NF & $31.7 \pm 5.9$ & $13.7 \pm 2.1$ & $2388 \pm 601^{\mathrm{b}}$ & 2 & 80 \\
AD10 & $35.5 \pm 4.5$ & $13.0 \pm 1.0$ & $1800 \pm 50^{\mathrm{b}}$ & 0 & 50 \\
AD12 & $51.2 \pm 3.5$ & $11.5 \pm 1.0$ & $683 \pm 136^{\mathrm{a}}$ & 1 & 80 \\
AD14 & $46.8 \pm 8.4$ & $11.0 \pm 0.6$ & $637 \pm 42^{\mathrm{a}}$ & 0 & 70 \\
AD10+NF & $42.0 \pm 6.2$ & $10.8 \pm 0.7$ & $1715 \pm 996^{\mathrm{b}}$ & 1 & 70 \\
AD12+NF & $55.7 \pm 2.7$ & $9.7 \pm 0.3$ & $2603 \pm 621^{\mathrm{b}}$ & 1 & 100 \\
AD14+NF & $54.4 \pm 11.1$ & $10.7 \pm 1.7$ & $1877 \pm 212^{\mathrm{b}}$ & 1 & 90 \\
\hline
\end{tabular}

Means ( \pm SEM) with the same letter within the column are not significantly different $(P<0.05)$.

*No significant differences among treatments. 
Table 5 Total lipids, neutral (NL) and polar (PL) lipid class contents ( $\mathrm{mg} \mathrm{g}^{-1} \mathrm{dry}$ weight) in newly hatched zoeae and ovaries of mud crab Scylla serrata fed artificial diets (AD) containing graded total lipid levels with or without natural food (NF)

\begin{tabular}{|c|c|c|c|c|c|c|c|}
\hline & \multirow[b]{2}{*}{ Control } & \multicolumn{3}{|l|}{$A D$} & \multicolumn{3}{|l|}{$A D+N F$} \\
\hline & & AD10 & AD12 & AD14 & $\mathrm{AD10}+\mathrm{NF}$ & $A D 12+N F$ & $\mathrm{AD} 14+\mathrm{NF}$ \\
\hline \multicolumn{8}{|l|}{ Zoeae } \\
\hline Total lipids & $143.37^{\circ}$ & $93.75^{\dagger}$ & $151.65^{d}$ & $171.25^{b}$ & $145.87^{\circ}$ & $161.83^{c}$ & $246.88^{a}$ \\
\hline Total NL & $91.18^{c}$ & $55.22^{t}$ & $81.53^{d}$ & $90.36^{c}$ & $76.28^{e}$ & $93.87^{b}$ & $131.97^{\mathrm{a}}$ \\
\hline $\mathrm{CHO}$ esters & $7.51^{d}$ & $0.63^{t}$ & $3.02^{e}$ & $3.05^{e}$ & $9.46^{c}$ & $10.42^{b}$ & $13.67^{a}$ \\
\hline TAG & $15.70^{\circ}$ & $10.97^{t}$ & $26.41^{d}$ & $33.29^{b}$ & $30.82^{c}$ & $33.50^{\mathrm{b}}$ & $43.67^{a}$ \\
\hline FFA & $35.39^{\mathrm{a}}$ & $18.61^{\theta}$ & $21.23^{d}$ & $23.21^{c}$ & $13.36^{t}$ & $21.85^{d}$ & $28.21^{b}$ \\
\hline $\mathrm{CHO}+\mathrm{DAG}$ & $25.30^{\circ}$ & $22.26^{d}$ & $22.70^{d}$ & $26.97^{b}$ & $17.37^{\circ}$ & $26.37^{b}$ & $40.20^{a}$ \\
\hline Total PL & $52.19^{t}$ & $38.53^{9}$ & $70.12^{c}$ & $80.89^{b}$ & $67.96^{\theta}$ & $69.59^{d}$ & $114.91^{a}$ \\
\hline$P E+P A$ & $8.30^{\circ}$ & $8.94^{d}$ & $16.33^{b}$ & $17.64^{\mathrm{a}}$ & $8.07^{\mathrm{e}}$ & $8.84^{d}$ & $11.76^{c}$ \\
\hline$P S+P I$ & $2.03^{b}$ & $0.45^{d}$ & $0.61^{d}$ & $1.74^{c}$ & $0.73^{d}$ & $0.63^{d}$ & $6.16^{a}$ \\
\hline PC & $18.67^{9}$ & $20.58^{e}$ & $32.89^{\circ}$ & $48.98^{b}$ & $40.57^{d}$ & $41.53^{c}$ & $62.94^{\mathrm{a}}$ \\
\hline SPH & $5.91^{\circ}$ & $3.21^{d}$ & $5.22^{\mathrm{c}}$ & $5.45^{\mathrm{c}}$ & $3.02^{d}$ & $6.46^{\mathrm{b}}$ & $8.83^{\mathrm{a}}$ \\
\hline LPC & $3.12^{\circ}$ & $2.92^{d}$ & $3.73^{c}$ & $1.41^{\theta}$ & $2.53^{d}$ & $5.37^{b}$ & $13.53^{a}$ \\
\hline \multicolumn{8}{|l|}{ Ovaries } \\
\hline Total lipids & $312.30^{c}$ & $226.20^{f}$ & $259.40^{\ominus}$ & $292.30^{d}$ & $253.50^{\ominus}$ & $327.40^{b}$ & $352.40^{\mathrm{a}}$ \\
\hline Total NL & $165.84^{\circ}$ & $117.87^{\dagger}$ & $147.44^{\mathrm{e}}$ & $162.77^{d}$ & $128.83^{9}$ & $170.27^{b}$ & $181.41^{a}$ \\
\hline $\mathrm{CHO}$ esters & $13.89^{\circ}$ & $7.91^{9}$ & $17.99^{d}$ & $26.74^{c}$ & $10.12^{t}$ & $32.49^{b}$ & $33.28^{a}$ \\
\hline TAG & $113.05^{a}$ & $73.65^{d}$ & $78.74^{\circ}$ & $84.64^{b}$ & $65.71^{9}$ & $67.39^{f}$ & $69.46^{e}$ \\
\hline FFA & $13.21^{t}$ & $16.63^{e}$ & $31.05^{c}$ & $31.98^{\mathrm{c}}$ & $30.12^{d}$ & $35.47^{b}$ & $38.04^{a}$ \\
\hline $\mathrm{CHO}+\mathrm{DAG}$ & $19.60^{\circ}$ & $14.81^{\theta}$ & $14.78^{\mathrm{B}}$ & $14.26^{\ominus}$ & $17.84^{\mathrm{d}}$ & $23.80^{\mathrm{b}}$ & $38.81^{a}$ \\
\hline Total PL & $146.46^{c}$ & $108.33^{9}$ & $111.96^{\prime}$ & $129.53^{d}$ & $124.67^{\mathrm{e}}$ & $157.13^{b}$ & $170.99^{\mathrm{a}}$ \\
\hline$P E+P A$ & $9.67^{\mathrm{c}}$ & $5.82^{\circ}$ & $8.75^{d}$ & $9.07^{d}$ & $8.72^{d}$ & $13.65^{b}$ & $26.03^{a}$ \\
\hline$P S+P I$ & $1.63^{\mathrm{a}}$ & $0.10^{d}$ & $1.27^{a}$ & $1.36^{\mathrm{a}}$ & $0.99^{c}$ & $0.96^{c}$ & $1.16^{\mathrm{b}}$ \\
\hline PC & $122.08^{c}$ & $87.79^{9}$ & $97.27^{\prime}$ & $103.97^{\ominus}$ & $108.11^{d}$ & $125.88^{b}$ & $128.23^{a}$ \\
\hline $\mathrm{SPH}$ & $4.06^{\mathrm{d}}$ & $1.43^{\mathrm{e}}$ & $6.04^{c}$ & $8.62^{b}$ & $1.76^{e}$ & $8.35^{\mathrm{b}}$ & $12.41^{a}$ \\
\hline LPC & $1.61^{\mathrm{a}}$ & $1.27^{\mathrm{a}}$ & $1.04^{\mathrm{a}}$ & $1.48^{a}$ & $1.71^{\mathrm{a}}$ & $1.39^{\mathrm{a}}$ & $1.99^{\mathrm{a}}$ \\
\hline
\end{tabular}

Means in the same row with the same superscripts are not significantly different at $P<0.05$.

See Table 2 for abbreviations.

several times higher than PL of which the main components were TAG, FFA and CHO+DAG. These components increased with dietary levels (Table 6). In muscles, PL was higher than NL and this was mainly composed of PC that increased correspondingly with dietary lipids. Crabs fed $\mathrm{AD}+\mathrm{NF}$ gave higher levels than crabs fed AD alone (Table 6).

Fatty acid composition and the HUFA concentrations in zoeae of $S$. serrata were significantly influenced by broodstock diets. The major fatty acid contents particularly 16:0, 18:0 and 18:1n-9, and the essential fatty acids ArA, EPA and DHA, were higher in zoeae of crabs fed $\mathrm{AD}+\mathrm{NF}$ than those fed $\mathrm{AD}$ only and these fatty acids increased corresponding to broodstock dietary lipid levels (Table 7). Arachidonic acid, EPA and DHA in zoeae from crabs fed AD+NF were higher than those of zoeae from crabs fed $A D$ alone. The $n-3 / n-6$ fatty acid ratios in zoeae from NF alone (control), $\mathrm{AD}$ and $\mathrm{AD}+\mathrm{NF}$ were 2.5, 2.3-1.9 and 2.2-2.5 respectively. Dominant fatty acids in the ovaries were 16:0, 16:In-7, 18:0, 18:1n-9, 18:1n-7, ArA,
EPA and DHA that increased with dietary lipids, higher levels in $\mathrm{AD}+\mathrm{NF}$ than in $\mathrm{AD}$ alone (Table 7). The n-3/n-6 fatty acid ratios in NF was 2.4 , in AD were 1.5-1.7 while in $\mathrm{AD}+\mathrm{NF}$ were 1.5-2.0. The $\mathrm{n}-3$ HUFA in hepatopancreas also increased with increasing dietary lipids, $\mathrm{AD}+\mathrm{NF}$ gave higher values than $\mathrm{AD}$ alone, with $\mathrm{n}-3 / \mathrm{n}-6$ fatty acid ratios in $\mathrm{AD}+\mathrm{NF}$ (1.2-1.7) higher than AD alone (0.9-1.0) (Table 8 ). The n-3 HUFA in muscles increased with dietary lipids of which the n-3/n- 6 fatty acid ratios from $\mathrm{NF}$ was 2.2, from $\mathrm{AD}$ were $1.8-2.2$ while for $\mathrm{AD}+\mathrm{NF}$ were 2.4-3.0 (Table 8).

\section{Discussion}

Broodstock nutrition had a considerable effect on the larval production and quality of $S$. serrata. Natural food alone or in combination with $\mathrm{AD}$ promoted higher larval production than $\mathrm{AD}$ alone. Larval quality of S. serrata in terms of total lipids, lipid classes and essential fatty acid contents improved by feeding 
Table 6 Total lipids, neutral (NL) and polar (PL) lipid class contents ( $\mathrm{mg} \mathrm{g}^{-1}$ dry weight) in hepatopancreas and muscle of mud crab Scylla serrata fed artificial diets (AD) containing graded total lipid levels with or without natural food (NF)

\begin{tabular}{|c|c|c|c|c|c|c|c|}
\hline & \multirow[b]{2}{*}{ Control } & \multicolumn{3}{|l|}{$A D$} & \multicolumn{3}{|l|}{$A D+N F$} \\
\hline & & AD10 & AD12 & AD14 & $A D 10+N F$ & $\mathrm{AD} 12+\mathrm{NF}$ & AD14+NF \\
\hline \multicolumn{8}{|l|}{ Hepatopancreas } \\
\hline Total lipids & $376.10^{t}$ & $353.90^{9}$ & $383.10^{\circ}$ & $391.00^{d}$ & $411.20^{\circ}$ & $449.90^{b}$ & $468.20^{a}$ \\
\hline Total NL & $343.08^{d}$ & $311.45^{t}$ & $335.73^{e}$ & $337.22^{\mathrm{e}}$ & $364.52^{\mathrm{c}}$ & $422.65^{b}$ & $449.33^{a}$ \\
\hline $\mathrm{CHO}$ esters & $8.02^{f}$ & $9.33^{e}$ & $13.84^{b}$ & $11.86^{c}$ & $15.84^{a}$ & $10.03^{d}$ & $4.77^{\mathrm{g}}$ \\
\hline TAG & $156.33^{t}$ & $141.51^{g}$ & $176.37^{\circ}$ & $183.13^{d}$ & $234.95^{c}$ & $286.85^{b}$ & $306.10^{\mathrm{a}}$ \\
\hline FFA & $120.50^{a}$ & $91.54^{b}$ & $87.29^{c}$ & $84.20^{d}$ & $67.51^{9}$ & $69.63^{\prime}$ & $72.78^{e}$ \\
\hline $\mathrm{CHO}+\mathrm{DAG}$ & $46.97^{\circ}$ & $46.58^{\circ}$ & $48.40^{d}$ & $52.07^{\circ}$ & $41.37^{f}$ & $57.50^{b}$ & $63.44^{\mathrm{a}}$ \\
\hline Total PL & $33.02^{\theta}$ & $42.45^{d}$ & $47.37^{b}$ & $53.78^{a}$ & $46.68^{c}$ & $27.25^{t}$ & $18.87^{9}$ \\
\hline$P E+P A$ & $2.35^{9}$ & $11.36^{\circ}$ & $10.70^{d}$ & $12.81^{b}$ & $19.72^{\mathrm{a}}$ & $5.32^{f}$ & $6.56^{\mathrm{e}}$ \\
\hline$P S+P I$ & $0.68^{d}$ & $0.50^{d}$ & $1.91^{\mathrm{b}}$ & $2.12^{a}$ & $1.25^{\mathrm{c}}$ & $1.27^{\circ}$ & $0.32^{\circ}$ \\
\hline PC & $17.30^{c}$ & $11.75^{t}$ & $21.20^{b}$ & $26.02^{a}$ & $14.94^{d}$ & $12.40^{e}$ & $7.39^{9}$ \\
\hline LPC & $3.81^{d}$ & $4.27^{\circ}$ & $5.78^{b}$ & $8.45^{\mathrm{a}}$ & $4.29^{\circ}$ & $1.97^{\mathrm{e}}$ & $0.58^{\prime}$ \\
\hline \multicolumn{8}{|l|}{ Muscle } \\
\hline Total lipids & $45.10^{\mathrm{a}}$ & $28.00^{9}$ & $31.80^{f}$ & $32.80^{\circ}$ & $38.10^{d}$ & $40.50^{c}$ & $42.60^{b}$ \\
\hline Total NL & $15.01^{\mathrm{a}}$ & $10.75^{\mathrm{b}}$ & $8.02^{\circ}$ & $7.62^{d}$ & $8.13^{c}$ & $6.69^{\ominus}$ & $6.97^{\circ}$ \\
\hline $\mathrm{CHO}$ esters & $1.78^{\mathrm{b}}$ & $1.49^{c}$ & $1.58^{\mathrm{C}}$ & $1.55^{\mathrm{c}}$ & $2.06^{a}$ & $0.50^{d}$ & $0.10^{\ominus}$ \\
\hline TAG & $1.32^{b}$ & $1.04^{\mathrm{c}}$ & $1.15^{\mathrm{c}}$ & $1.54^{\mathrm{b}}$ & $0.04^{d}$ & $1.10^{\mathrm{C}}$ & $2.16^{a}$ \\
\hline FFA & $5.48^{\mathrm{a}}$ & $0.52^{b}$ & $0.29^{d}$ & $0.26^{d}$ & $0.50^{\mathrm{b}}$ & $0.10^{e}$ & $0.41^{c}$ \\
\hline $\mathrm{CHO}+\mathrm{DAG}$ & $5.78^{b}$ & $6.41^{a}$ & $4.63^{\mathrm{C}}$ & $4.07^{\circ}$ & $4.35^{c}$ & $3.95^{\mathrm{d}}$ & $2.96^{\mathrm{e}}$ \\
\hline Total PL & $30.09^{c}$ & $17.25^{g}$ & $23.79^{t}$ & $25.18^{\circ}$ & $29.97^{d}$ & $33.81^{\mathrm{b}}$ & $35.63^{a}$ \\
\hline$P E+P A$ & $3.39^{\mathrm{c}}$ & $2.36^{\mathrm{d}}$ & $2.96^{d}$ & $3.77^{\mathrm{c}}$ & $4.50^{\mathrm{b}}$ & $5.31^{a}$ & $5.63^{\mathrm{a}}$ \\
\hline$P S+P I$ & $0.30^{a}$ & $0.07^{b}$ & $0.10^{\mathrm{b}}$ & $0.07^{b}$ & $0.25^{a}$ & $0.09^{b}$ & $0.27^{\mathrm{a}}$ \\
\hline PC & $20.83^{c}$ & $9.99^{f}$ & $16.20^{\mathrm{e}}$ & $16.34^{e}$ & $18.93^{d}$ & $21.93^{b}$ & $22.41^{a}$ \\
\hline $\mathrm{SPH}$ & $1.05^{d}$ & $3.88^{b}$ & $3.92^{\mathrm{b}}$ & $4.37^{a}$ & $3.25^{c}$ & $3.73^{b}$ & $4.06^{a}$ \\
\hline LPC & $1.50^{\mathrm{b}}$ & $0.10^{\circ}$ & $0.27^{d}$ & $0.10^{\mathrm{e}}$ & $0.85^{\mathrm{C}}$ & $1.88^{\mathrm{a}}$ & $1.87^{\mathrm{a}}$ \\
\hline
\end{tabular}

Means in the same row with the same superscripts are not significantly different at $P<0.05$.

See Table 2 for abbreviations.

broodstock crabs with NF in combination with $\mathrm{AD}$. In a separate study using the same batch of newly hatched zoeae, larval quality was evaluated based on their tolerance to formalin exposure at $0-15 \mathrm{ppm}$ for 24,48 and $72 \mathrm{~h}$. Survival was higher in larvae from broodstock fed AD+NF followed by NF and the lowest was from AD (J. de Pedro, unpubl. data).

For sub-adult $S$. serrata, AD containing $32-40 \%$ dietary protein, $6 \%$ or $12 \%$ lipid, $15-18 \mathrm{MJ} \mathrm{kg}^{-1} \mathrm{en}$ ergy and $20-28 \mathrm{mg}$ protein $\mathrm{kJ}^{-1} \mathrm{P} / \mathrm{E}$ ratio were found to enhance good growth (Catacutan 2002). The dietary $\mathrm{CHO}$ requirement of juvenile mud crab was about $0.51 \%$ (Sheen 2000) while the dietary lipid requirement was 5.3-13.8\% (cod liver oil/corn oil, 2:1 w/w) (Sheen \& Wu 1999). For S. serrata broodstock, NF alone and NF in combination with $\mathrm{AD}$ containing $42 \%$ protein and $10-14 \%$ lipid were found to enhance zoeae production in this study. This dietary lipid level was close or higher than that reported to maximize reproduction of Penaeus stylirostris (10.3$11.1 \%$ ) fed $60 \% \mathrm{AD}$ and $40 \%$ multiple fresh supplement (Bray et al. 1990).
Eyestalk ablation is commonly used to increase spawning frequency and to hasten the development of gonads in decapod crustaceans. Scylla serrata matures in captivity even without eyestalk ablation, but ablated females fed $\mathrm{AD}$ alone performed better than their intact counterparts (Millamena \& Quinitio 2000). However, strict hygiene protocol is needed when using $\mathrm{AD}$ alone because several problems such as higher number of melanized ovaries and brown patches on exoskeleton may be encountered (E.T. Quinitio, unpubl. data). Although it is possible to mature and spawn crab females fed either $\mathrm{NF}$ or $\mathrm{AD}$ alone, provision of essential lipid components in $\mathrm{AD}$ in combination with NF improved the production of better-quality zoeae that contained higher metabolic energy, phospholipids, essential fatty acids and other lipid materials required for survival and larval development before exogenous feeding. As documented in other crustaceans, a combination diet can provide the essential nutrients that are deficient in NF or vice versa to meet maternal requirements and for the production of viable larvae (Galgani, Goguenheim, 
Table 7 Fatty acid contents ( $\mathrm{mg} \mathrm{g}^{-1}$ dry weight) in newly hatched zoeae and ovaries of mud crab Scylla serrata fed artificial diets (AD) containing graded total lipid levels with or without natural food (NF)

\begin{tabular}{|c|c|c|c|c|c|c|c|c|c|c|c|c|c|c|}
\hline \multirow[b]{2}{*}{ FAME } & \multicolumn{7}{|l|}{ Zoeae } & \multicolumn{7}{|l|}{ Ovaries } \\
\hline & Control & AD10 & AD12 & AD14 & $\begin{array}{l}\text { AD10+ } \\
\text { NF }\end{array}$ & $\begin{array}{l}\text { AD12+ } \\
\text { NF }\end{array}$ & $\begin{array}{l}\text { AD14+ } \\
\text { NF }\end{array}$ & Control & AD10 & AD12 & AD14 & $\begin{array}{l}\text { AD10+ } \\
\text { NF }\end{array}$ & $\begin{array}{l}\text { AD12+ } \\
\text { NF }\end{array}$ & $\begin{array}{l}\text { AD14+ } \\
\text { NF }\end{array}$ \\
\hline 14:0 & $1.50^{\circ}$ & $1.03^{\mathrm{d}}$ & $1.49^{\circ}$ & $2.91^{\mathrm{a}}$ & $2.50^{\mathrm{b}}$ & $2.29^{b}$ & $2.72^{\mathrm{a}}$ & $6.91^{\mathrm{a}}$ & $5.28^{\mathrm{b}}$ & $4.96^{\circ}$ & $6.35^{\mathrm{a}}$ & $5.10^{\mathrm{bc}}$ & $5.80^{b}$ & $6.89^{\mathrm{a}}$ \\
\hline $15: 0$ & $1.02^{\mathrm{d}}$ & $0.71^{e}$ & $1.15^{\mathrm{d}}$ & $1.44^{\mathrm{c}}$ & $1.22^{\mathrm{d}}$ & $1.83^{b}$ & $1.96^{\mathrm{a}}$ & $2.99^{\mathrm{a}}$ & $1.66^{\mathrm{d}}$ & $2.18^{\mathrm{C}}$ & $2.56^{\mathrm{b}}$ & $1.88^{d}$ & $2.68^{\mathrm{b}}$ & $2.28^{\mathrm{bc}}$ \\
\hline $15: 1(n-5)$ & $0.54^{d}$ & $0.70^{\mathrm{d}}$ & $1.03^{b}$ & $0.85^{c}$ & $0.84^{c}$ & $1.11^{\mathrm{b}}$ & $1.60^{\mathrm{a}}$ & $1.73^{\mathrm{d}}$ & $1.41^{c}$ & $2.31^{b}$ & $2.10^{\mathrm{b}}$ & $1.42^{\circ}$ & $2.51^{\mathrm{ab}}$ & $2.73^{\mathrm{a}}$ \\
\hline 16:0 & $19.77^{t}$ & $16.29^{9}$ & $23.52^{\ominus}$ & $26.24^{c}$ & $24.73^{d}$ & $28.68^{\mathrm{b}}$ & $42.55^{a}$ & $60.48^{\mathrm{a}}$ & $43.25^{f}$ & $43.33^{t}$ & $52.60^{d}$ & $48.06^{\mathrm{e}}$ & $55.19^{\mathrm{c}}$ & $57.13^{\mathrm{a}}$ \\
\hline $16: 1(n-7)$ & $2.99^{d}$ & $2.17^{\mathrm{e}}$ & $3.29^{d}$ & $5.19^{b}$ & $6.25^{\mathrm{a}}$ & $4.31^{c}$ & $5.29^{b}$ & $21.38^{\mathrm{a}}$ & $10.97^{\mathrm{e}}$ & $11.01^{\mathrm{e}}$ & $13.63^{d}$ & $13.68^{d}$ & $14.13^{c}$ & $15.53^{b}$ \\
\hline $17: 0$ & $1.94^{\mathrm{d}}$ & $1.96^{\circ}$ & $2.73^{b}$ & $2.06^{c}$ & $1.83^{\mathrm{d}}$ & $2.96^{b}$ & $4.40^{\mathrm{a}}$ & $4.99^{\mathrm{a}}$ & $2.39^{\circ}$ & $3.11^{d}$ & $3.65^{\circ}$ & $2.95^{d}$ & $4.60^{\mathrm{b}}$ & $3.69^{c}$ \\
\hline 18:0 & $11.51^{t}$ & $9.83^{g}$ & $12.57^{\circ}$ & $14.20^{C}$ & $13.45^{\mathrm{d}}$ & $16.98^{\mathrm{b}}$ & $28.90^{\mathrm{a}}$ & $30.99^{\mathrm{a}}$ & $18.62^{\ominus}$ & $20.63^{d}$ & $22.82^{\circ}$ & $23.06^{\mathrm{C}}$ & $29.28^{\mathrm{b}}$ & $31.34^{\mathrm{a}}$ \\
\hline $18: 1(n-9)$ & $11.15^{t}$ & $10.52^{9}$ & $14.81^{\ominus}$ & $21.56^{\mathrm{b}}$ & $17.90^{d}$ & $18.60^{\circ}$ & $30.32^{\mathrm{a}}$ & $41.47^{b}$ & $38.87^{\circ}$ & $38.95^{\circ}$ & $42.71^{\mathrm{ab}}$ & $35.29^{d}$ & $42.89^{\mathrm{ab}}$ & $43.17^{\mathrm{a}}$ \\
\hline $18: 1(n-7)$ & $4.25^{d}$ & $3.53^{\ominus}$ & $4.93^{d}$ & $5.64^{c}$ & $5.16^{c}$ & $6.93^{\mathrm{b}}$ & $10.05^{a}$ & $11.85^{a}$ & $8.38^{d}$ & $8.69^{d}$ & $10.79^{b}$ & $9.44^{\mathrm{c}}$ & $11.06^{\mathrm{a}}$ & $11.84^{\mathrm{a}}$ \\
\hline $18: 2(n-6)-t$ & $0.23^{c}$ & $0.31^{\mathrm{a}}$ & $0.49^{\mathrm{a}}$ & $0.42^{b}$ & $0.43^{b}$ & $0.50^{\mathrm{a}}$ & $0.55^{\mathrm{a}}$ & $0.73^{\mathrm{b}}$ & $0.53^{c}$ & $0.52^{c}$ & $0.77^{b}$ & $0.57^{c}$ & $0.83^{\mathrm{a}}$ & $0.73^{b}$ \\
\hline $18: 2(n-6)-c$ & $1.13^{\mathrm{g}}$ & $2.04^{\prime}$ & $2.97^{\circ}$ & $10.10^{\mathrm{a}}$ & $3.31^{\mathrm{d}}$ & $4.60^{\circ}$ & $5.71^{\mathrm{b}}$ & $3.70^{\mathrm{g}}$ & $17.77^{\mathrm{a}}$ & $13.22^{\mathrm{c}}$ & $14.44^{b}$ & $8.72^{\prime}$ & $11.78^{d}$ & $9.84^{\mathrm{e}}$ \\
\hline $18: 3(n-3)$ & $0.62^{d}$ & $0.57^{d}$ & $1.14^{b}$ & $1.27^{\mathrm{a}}$ & $0.88^{\circ}$ & $1.01^{b}$ & $1.27^{\mathrm{a}}$ & $1.98^{\mathrm{b}}$ & $1.67^{\circ}$ & $2.11^{\mathrm{b}}$ & $2.10^{\mathrm{b}}$ & $1.44^{\mathrm{d}}$ & $2.68^{a}$ & $1.59^{\circ}$ \\
\hline $20: 4(n-6)$ & $6.68^{\circ}$ & $6.34^{\circ}$ & $8.46^{\circ}$ & $9.43^{b}$ & $7.43^{d}$ & $9.48^{b}$ & $16.35^{\mathrm{a}}$ & $14.32^{\mathrm{\theta}}$ & $7.27^{9}$ & $16.09^{d}$ & $18.00^{\circ}$ & $11.65^{\prime}$ & $24.34^{\mathrm{b}}$ & $35.61^{a}$ \\
\hline $20: 5(n-3)$ & $10.43^{\theta}$ & $10.36^{\ominus}$ & $12.72^{d}$ & $13.72^{\mathrm{c}}$ & $11.56^{\ominus}$ & $16.28^{\mathrm{b}}$ & $27.51^{a}$ & $18.70^{\circ}$ & $10.28^{t}$ & $14.94^{\theta}$ & $17.48^{\mathrm{d}}$ & $15.29^{\circ}$ & $20.23^{\mathrm{b}}$ & $29.90^{\mathrm{a}}$ \\
\hline 22:4(n-6) & $0.87^{c}$ & $0.72^{\mathrm{cd}}$ & $1.00^{\mathrm{b}}$ & $0.61^{d}$ & $1.01^{b}$ & $0.79^{c}$ & $1.48^{\mathrm{a}}$ & $2.91^{b}$ & $1.07^{f}$ & $1.63^{\mathrm{e}}$ & $2.00^{d}$ & $1.99^{d}$ & $4.18^{\mathrm{a}}$ & $2.27^{\mathrm{c}}$ \\
\hline $22: 5(n-6)$ & $1.11^{\mathrm{b}}$ & $0.76^{\mathrm{d}}$ & $1.03^{\circ}$ & $1.01^{\mathrm{c}}$ & $1.24^{\mathrm{b}}$ & $1.16^{\mathrm{b}}$ & $1.94^{\mathrm{a}}$ & $3.43^{b}$ & $1.96^{\mathrm{d}}$ & $2.98^{\circ}$ & $3.07^{\circ}$ & $2.99^{\mathrm{c}}$ & $3.44^{\mathrm{b}}$ & $3.83^{\mathrm{a}}$ \\
\hline $5(n-3)$ & $1.58^{\mathrm{c}}$ & $1.27^{\mathrm{d}}$ & $1.80^{\circ}$ & $2.62^{b}$ & $2.32^{\mathrm{b}}$ & $2.81^{b}$ & $3.23^{a}$ & $5.17^{\circ}$ & $3.21^{e}$ & $4.25^{d}$ & $6.44^{b}$ & $4.19^{d}$ & $7.63^{\mathrm{a}}$ & $4.95^{c}$ \\
\hline 22:6(n-3) & $13.41^{\mathrm{e}}$ & $10.90^{\prime}$ & $13.27^{e}$ & $22.37^{b}$ & $15.11^{d}$ & $17.39^{c}$ & $29.49^{\mathrm{a}}$ & $33.35^{\mathrm{d}}$ & $26.31^{\prime}$ & $35.12^{\mathrm{bc}}$ & $35.69^{b}$ & $32.15^{\ominus}$ & $34.67^{\circ}$ & $42.66^{a}$ \\
\hline Sum saturates & $37.76^{d}$ & $31.10^{\circ}$ & $46.06^{\circ}$ & $46.38^{\circ}$ & $45.82^{\circ}$ & $55.58^{\mathrm{b}}$ & $83.70^{\mathrm{a}}$ & $109.54^{\mathrm{a}}$ & $72.91^{9}$ & $76.86^{t}$ & $90.78^{d}$ & $83.14^{\ominus}$ & $102.46^{\circ}$ & $106.45^{\mathrm{b}}$ \\
\hline Sum monoenes & $24.22^{\mathrm{e}}$ & $20.79^{\prime}$ & $31.95^{\mathrm{d}}$ & $41.65^{\mathrm{bc}}$ & $38.81^{\circ}$ & $39.99^{\mathrm{b}}$ & $56.14^{a}$ & $92.21^{\mathrm{a}}$ & $68.94^{t}$ & $71.35^{d}$ & $80.97^{\circ}$ & $69.91^{\mathrm{B}}$ & $86.29^{b}$ & $86.91^{b}$ \\
\hline Sum n-3 FA & $27.25^{\ominus}$ & $24.21^{t}$ & $30.83^{d}$ & $41.76^{b}$ & $31.10^{\circ}$ & $39.56^{\mathrm{c}}$ & $64.22^{\mathrm{a}}$ & $62.42^{\mathrm{d}}$ & $42.70^{9}$ & $58.90^{\circ}$ & $63.94^{\mathrm{c}}$ & $54.51^{f}$ & $69.16^{\mathrm{b}}$ & $80.63^{a}$ \\
\hline Sum n-6 FA & $10.53^{\mathrm{e}}$ & $10.63^{\mathrm{e}}$ & $14.57^{d}$ & $22.08^{\mathrm{b}}$ & $14.12^{\mathrm{d}}$ & $17.20^{\circ}$ & $26.20^{\mathrm{a}}$ & $26.74^{e}$ & $29.47^{d}$ & $37.33^{\mathrm{c}}$ & $37.48^{\mathrm{C}}$ & $27.11^{\mathrm{e}}$ & $46.30^{\mathrm{b}}$ & $53.57^{\mathrm{a}}$ \\
\hline Sum n-3 HUFA & $26.37^{\mathrm{e}}$ & $23.35^{t}$ & $29.29^{d}$ & $39.62^{b}$ & $29.94^{d}$ & $37.69^{\mathrm{c}}$ & $62.70^{\mathrm{a}}$ & $59.58^{\mathrm{d}}$ & $40.69^{9}$ & $56.21^{\mathrm{e}}$ & $61.34^{\circ}$ & $52.59^{f}$ & $65.59^{\mathrm{b}}$ & $78.60^{\mathrm{a}}$ \\
\hline Ratio $n-3 / n-6$ FA & $2.54^{\mathrm{a}}$ & $2.28^{\mathrm{b}}$ & $2.11^{\mathrm{c}}$ & $1.89^{d}$ & $2.17^{c}$ & $2.30^{\mathrm{b}}$ & $2.45^{\mathrm{a}}$ & $2.36^{a}$ & $1.45^{\mathrm{e}}$ & $1.57^{\circ}$ & $1.71^{\circ}$ & $2.01^{\mathrm{b}}$ & $1.49^{\mathrm{d}}$ & $1.51^{\mathrm{d}}$ \\
\hline
\end{tabular}

Means in the same row with the same superscripts under zoeae or ovaries are not significantly different at $P<0.05$.

FAME, fatty acid methyl esters; HUFA, highly unsaturated fatty acids.

Galgani \& Cuzon 1989; Millamena 1989; Nascimento, Bray, Leung Trujillo \& Lawrence 1991; Millamena \& Quinitio 2000).

One indication of the dietary lipid requirement of an animal can be derived from its tissue composition. Mature $S$. serrata ovaries have been shown to contain a large total lipid component, $33.8 \% \mathrm{DW}$ for wild and $45.0 \%$ DW for pond-sourced crabs (Alava et al. 2007). These were higher than those obtained from the present study in which the ovarian total lipid contents of $\mathrm{NF}$ (control), $\mathrm{AD}$ and $\mathrm{AD}+\mathrm{NF}$ were $31.2 \%, 22.6-$ $29.2 \%$ and $25.5-35.2 \%$ DW respectively (Table 5). Moreover, the hepatopancreas of mature S. serrata contained high total lipids, $43.0 \%$ DW for wild and 44.3\% DW for pond-reared crabs (Alava et al. 2007). Total lipids were high in the hepatopancreas from all the dietary treatments, 35.4-39.1\% DW. Total lipids in crab muscle ranged from $2.8 \%$ to $4.5 \%$ DW (Table 6) and these were either lower or close to those of muscle TL of wild (4.8\%) and pond-reared (4.3\% DW) mature crabs (Alava et al. 2007). These data indicate that
S. serrata has a high capacity for tissue lipid deposition. The tissue lipid composition of this species was influenced by dietary lipid as reported for penaeid spawners (Cahu \& Quazuguel 1989; Cahu et al. 1994).

The total lipid level in newly hatched zoeae from crabs fed control diet was $14.3 \%$, zoeae from broodstock fed AD10, AD12 and AD14 were 9.4\%, 15.2\% and $20.1 \%$ and zoeae from $\mathrm{AD} 10+\mathrm{NF}, \mathrm{AD} 12+\mathrm{NF}$ and $\mathrm{AD} 14+\mathrm{NF}$ were $14.6 \%, 16.2 \%$ and $24.7 \% \mathrm{DW}$ (Table 5). These levels were higher than those of the newly hatched zoeae $(8.89 \%$ DW) from pond-reared crabs that were fed fish biomass and small bivalves (V. R. Alava, unpubl. data). Phospholipids, particularly PC, accumulated in large amounts in the ovaries and were mobilized to the newly spawned eggs of S. serrata (Alava et al. 2007). Except for AD10 diet, the phospholipid levels in the newly hatched zoeae (Table 6) were close or even higher than those in zoeae from pond-reared mature crabs $(5.7 \% \mathrm{DW})$ (V. R. Alava, unpubl. data). These results indicated that the dietary treatments provided sufficient lipid reserves essential 
Table 8 Fatty acid contents ( $\mathrm{mg} \mathrm{g}^{-1}$ dry weight) in hepatopancreas and muscle of mud crab Scylla serrata fed artificial diets (AD) containing graded total lipid levels with or without natural food (NF)

\begin{tabular}{|c|c|c|c|c|c|c|c|c|c|c|c|c|c|c|}
\hline \multirow[b]{2}{*}{ FAME } & \multicolumn{7}{|c|}{ Hepatopancreas } & \multicolumn{7}{|l|}{ Muscle } \\
\hline & Control & AD10 & AD12 & AD14 & $\begin{array}{l}\text { AD10+ } \\
\text { NF }\end{array}$ & $\begin{array}{l}\text { AD12+ } \\
\text { NF }\end{array}$ & $\begin{array}{l}\text { AD14+ } \\
\text { NF }\end{array}$ & Control & AD10 & AD12 & AD14 & $\begin{array}{l}\text { AD10+ } \\
\text { NF }\end{array}$ & $\begin{array}{l}\text { AD12+ } \\
\text { NF }\end{array}$ & $\begin{array}{l}\text { AD14+ } \\
\text { NF }\end{array}$ \\
\hline $14: 0$ & $12.64^{a}$ & $12.87^{\mathrm{a}}$ & $8.53^{d}$ & $10.91^{\circ}$ & $10.10^{c}$ & $11.26^{b}$ & $10.58^{c}$ & $0.56^{\mathrm{a}}$ & $0.13^{d}$ & $0.23^{b}$ & $0.17^{d}$ & $0.23^{\mathrm{c}}$ & $0.18^{\circ}$ & $0.24^{b}$ \\
\hline $15: 0$ & $4.57^{b}$ & $3.59^{\circ}$ & $2.51^{d}$ & $3.26^{c}$ & $4.36^{\mathrm{b}}$ & $7.07^{\mathrm{a}}$ & $3.77^{\mathrm{c}}$ & $0.25^{\mathrm{a}}$ & $0.07^{e}$ & $0.09^{\circ}$ & $0.13^{d}$ & $0.12^{b}$ & $0.15^{\circ}$ & $0.16^{\mathrm{b}}$ \\
\hline $16: 0$ & $92.19^{\mathrm{c}}$ & $79.31^{t}$ & $80.46^{f}$ & $90.08^{d}$ & $82.09^{\mathrm{e}}$ & $97.24^{\mathrm{b}}$ & $101.53^{\mathrm{a}}$ & $5.76^{\mathrm{a}}$ & $1.57^{\circ}$ & $2.32^{\mathrm{c}}$ & $2.72^{d}$ & $2.72^{b}$ & $3.10^{c}$ & $3.49^{\mathrm{b}}$ \\
\hline $16: 1(n-7)$ & $21.11^{\mathrm{a}}$ & $13.14^{\circ}$ & $16.46^{d}$ & $17.67^{\circ}$ & $19.48^{\mathrm{b}}$ & $19.66^{\mathrm{b}}$ & $17.91^{c}$ & $1.66^{\mathrm{a}}$ & $0.54^{\circ}$ & $0.65^{\circ}$ & $0.90^{d}$ & $0.95^{b}$ & $1.13^{c}$ & $1.49^{\mathrm{a}}$ \\
\hline $17: 0$ & $6.91^{\mathrm{b}}$ & $5.15^{\mathrm{c}}$ & $5.48^{\mathrm{c}}$ & $5.49^{c}$ & $7.22^{\mathrm{b}}$ & $8.27^{\mathrm{a}}$ & $7.05^{b}$ & $0.51^{\mathrm{a}}$ & $0.23^{\circ}$ & $0.25^{c}$ & $0.29^{c}$ & $0.29^{b}$ & $0.35^{c}$ & $0.40^{\mathrm{b}}$ \\
\hline $18: 0$ & $37.69^{d}$ & $26.04^{f}$ & $25.58^{f}$ & $34.35^{8}$ & $39.90^{\mathrm{c}}$ & $42.06^{\mathrm{b}}$ & $44.42^{a}$ & $3.72^{\mathrm{a}}$ & $1.95^{d}$ & $2.20^{\circ}$ & $2.65^{\circ}$ & $2.42^{b}$ & $2.72^{b}$ & $2.86^{b}$ \\
\hline $18: 1(n-9)$ & $34.37^{9}$ & $60.55^{c}$ & $63.88^{b}$ & $65.64^{\mathrm{a}}$ & $47.08^{t}$ & $57.61^{d}$ & $51.96^{c}$ & $4.27^{a}$ & $2.30^{d}$ & $3.31^{c}$ & $3.80^{c}$ & $3.23^{b}$ & $4.07^{c}$ & $3.45^{\mathrm{c}}$ \\
\hline $18: 1(n-7)$ & $17.61^{c}$ & $15.53^{\mathrm{e}}$ & $16.17^{d}$ & $18.45^{b}$ & $16.90^{d}$ & $21.72^{a}$ & $21.13^{a}$ & $1.09^{\mathrm{a}}$ & $0.34^{\theta}$ & $0.50^{c}$ & $0.65^{d}$ & $0.63^{b}$ & $0.71^{\mathrm{c}}$ & $0.77^{b}$ \\
\hline $18: 2(n-6)-t$ & $1.32^{\mathrm{cd}}$ & $1.10^{\theta}$ & $1.59^{c}$ & $1.62^{b}$ & $1.25^{d}$ & $1.70^{\mathrm{b}}$ & $2.20^{\mathrm{a}}$ & $0.08^{a}$ & $0.05^{a}$ & $0.06^{\mathrm{a}}$ & $0.06^{\mathrm{a}}$ & $0.05^{a}$ & $0.06^{a}$ & $0.05^{\mathrm{a}}$ \\
\hline $18: 2(n-6)-c$ & $4.85^{g}$ & $30.64^{b}$ & $33.41^{\mathrm{a}}$ & $25.30^{\circ}$ & $9.28^{t}$ & $13.87^{e}$ & $20.77^{d}$ & $0.82^{d}$ & $0.43^{t}$ & $1.26^{\mathrm{a}}$ & $1.17^{b}$ & $0.70^{e}$ & $1.04^{b c}$ & $0.93^{c}$ \\
\hline $18: 3(n-3)$ & $1.97^{\mathrm{c}}$ & $2.97^{\mathrm{a}}$ & $2.85^{\mathrm{a}}$ & $2.14^{\mathrm{c}}$ & $2.22^{\mathrm{b}}$ & $2.26^{\mathrm{b}}$ & $2.63^{\mathrm{ab}}$ & $0.24^{\mathrm{a}}$ & $0.09^{c}$ & $0.19^{b}$ & $0.20^{\mathrm{ab}}$ & $0.17^{a}$ & $0.25^{a}$ & $0.27^{\mathrm{a}}$ \\
\hline $18: 4(n-3)$ & $1.01^{\mathrm{a}}$ & $0.72^{\mathrm{a}}$ & $0.67^{\mathrm{a}}$ & $0.49^{\mathrm{a}}$ & $0.71^{a}$ & $0.83^{\mathrm{a}}$ & $0.79^{\mathrm{a}}$ & $0.07^{a}$ & $0.03^{a}$ & $0.04^{\mathrm{a}}$ & $0.04^{\mathrm{a}}$ & $0.06^{a}$ & $0.07^{\mathrm{a}}$ & $0.09^{\mathrm{a}}$ \\
\hline $20: 0$ & $3.25^{\mathrm{ab}}$ & $2.93^{\mathrm{c}}$ & $3.11^{\mathrm{b}}$ & $3.68^{\mathrm{a}}$ & $2.86^{\mathrm{c}}$ & $3.77^{\mathrm{a}}$ & $3.87^{\mathrm{a}}$ & $0.26^{\mathrm{a}}$ & $0.16^{\mathrm{b}}$ & $0.11^{b}$ & $0.14^{b}$ & $0.12^{b}$ & $0.13^{b}$ & $0.10^{b}$ \\
\hline $20: 1(n-9)$ & $6.34^{d}$ & $7.67^{\mathrm{C}}$ & $17.61^{\mathrm{a}}$ & $13.12^{b}$ & $7.27^{\circ}$ & $13.21^{b}$ & $16.94^{a}$ & $0.57^{\mathrm{a}}$ & $0.07^{d}$ & $0.22^{b}$ & $0.21^{b}$ & $0.17^{c}$ & $0.20^{b}$ & $0.20^{\mathrm{b}}$ \\
\hline $20: 1(n-7)$ & $3.97^{\mathrm{a}}$ & $1.95^{\mathrm{d}}$ & $2.45^{d}$ & $2.40^{d}$ & $3.02^{c}$ & $3.68^{b}$ & $4.31^{\mathrm{a}}$ & $0.18^{\mathrm{a}}$ & $0.01^{b}$ & $0.02^{b}$ & $0.02^{b}$ & $0.03^{b}$ & $0.03^{b}$ & $0.04^{b}$ \\
\hline $20: 3(n-6)$ & $1.59^{\mathrm{a}}$ & $0.61^{d}$ & $0.52^{\circ}$ & $0.87^{\mathrm{c}}$ & $0.80^{\circ}$ & $1.42^{\mathrm{b}}$ & $1.41^{\mathrm{b}}$ & $0.08^{a}$ & $0.01^{a}$ & $0.02^{\mathrm{a}}$ & $0.03^{\mathrm{a}}$ & $0.03^{a}$ & $0.03^{a}$ & $0.03^{\mathrm{a}}$ \\
\hline $20: 4(n-6)$ & $14.26^{\mathrm{a}}$ & $6.14^{\mathrm{c}}$ & $6.33^{\mathrm{c}}$ & $12.98^{b}$ & $13.99^{\mathrm{ab}}$ & $13.32^{b}$ & $13.33^{b}$ & $2.14^{\mathrm{a}}$ & $2.03^{a}$ & $1.81^{\circ}$ & $2.12^{a}$ & $1.87^{\circ}$ & $1.97^{\mathrm{b}}$ & $1.93^{b}$ \\
\hline $20: 3(n-3)$ & $1.01^{\mathrm{ab}}$ & $0.64^{\mathrm{c}}$ & $0.61^{c}$ & $0.26^{d}$ & $0.46^{\mathrm{c}}$ & $1.22^{\mathrm{a}}$ & $0.93^{\mathrm{ab}}$ & $0.06^{\mathrm{a}}$ & $0.03^{\mathrm{a}}$ & $0.03^{\mathrm{a}}$ & $0.03^{a}$ & $0.04^{\mathrm{a}}$ & $0.06^{\mathrm{a}}$ & $0.05^{\mathrm{a}}$ \\
\hline $20: 5(n-3)$ & $14.20^{\mathrm{b}}$ & $7.38^{f}$ & $8.46^{\circ}$ & $9.76^{d}$ & $23.06^{\mathrm{a}}$ & $13.88^{\circ}$ & $14.98^{\mathrm{b}}$ & $3.71^{\mathrm{b}}$ & $2.59^{\circ}$ & $3.42^{b}$ & $3.97^{\mathrm{b}}$ & $3.47^{b}$ & $4.26^{\mathrm{a}}$ & $4.66^{a}$ \\
\hline $22: 1(n-9)$ & $1.09^{d}$ & $1.60^{\circ}$ & $3.11^{a b}$ & $2.67^{b}$ & $1.47^{\mathrm{c}}$ & $3.06^{\mathrm{ab}}$ & $3.52^{\mathrm{a}}$ & $0.14^{\mathrm{a}}$ & $0.06^{\mathrm{b}}$ & $0.04^{b}$ & $0.05^{b}$ & $0.05^{b}$ & $0.06^{b}$ & $0.05^{b}$ \\
\hline $22: 4(n-6)$ & $4.00^{\mathrm{a}}$ & $1.12^{\theta}$ & $0.88^{\ominus}$ & $1.62^{d}$ & $2.22^{\circ}$ & $3.40^{\mathrm{b}}$ & $3.09^{b}$ & $0.19^{\mathrm{a}}$ & $0.06^{b}$ & $0.06^{\mathrm{b}}$ & $0.08^{b}$ & $0.09^{b}$ & $0.09^{b}$ & $0.09^{b}$ \\
\hline $22: 5(n-6)$ & $4.87^{\mathrm{a}}$ & $2.50^{\mathrm{b}}$ & $2.70^{\mathrm{b}}$ & $2.84^{b}$ & $2.74^{\mathrm{b}}$ & $4.48^{a}$ & $4.50^{\mathrm{a}}$ & $0.31^{a}$ & $0.12^{\mathrm{d}}$ & $0.15^{\mathrm{bc}}$ & $0.16^{c}$ & $0.18^{b}$ & $0.19^{b}$ & $0.20^{\mathrm{b}}$ \\
\hline $22: 5(n-3)$ & $4.18^{c}$ & $2.70^{\ominus}$ & $3.64^{d}$ & $3.82^{d}$ & $3.30^{d}$ & $6.28^{\mathrm{a}}$ & $5.31^{b}$ & $0.33^{\mathrm{a}}$ & $0.12^{\mathrm{c}}$ & $0.17^{b}$ & $0.28^{\mathrm{b}}$ & $0.22^{\mathrm{a}}$ & $0.30^{\mathrm{ab}}$ & $0.23^{b}$ \\
\hline $22: 6(n-3)$ & $25.89^{\mathrm{c}}$ & $21.64^{d}$ & $26.09^{c}$ & $26.62^{c}$ & $25.64^{\mathrm{c}}$ & $28.29^{b}$ & $31.16^{\mathrm{a}}$ & $3.62^{b}$ & $1.85^{\circ}$ & $3.20^{\mathrm{b}}$ & $3.54^{\mathrm{b}}$ & $3.05^{\mathrm{ab}}$ & $4.08^{b}$ & $4.46^{\mathrm{a}}$ \\
\hline Sum saturates & $164.77^{c}$ & $134.88^{e}$ & $135.66^{e}$ & $154.71^{d}$ & $151.97^{d}$ & $174.42^{b}$ & $181.12^{\mathrm{a}}$ & $11.40^{\mathrm{a}}$ & $4.30^{\mathrm{e}}$ & $5.22^{d}$ & $6.19^{c}$ & $5.97^{d}$ & $6.71^{c}$ & $7.34^{b}$ \\
\hline Sum monoenes & $99.08^{\theta}$ & $113.44^{\circ}$ & $124.04^{b}$ & $133.56^{\mathrm{a}}$ & $107.04^{d}$ & $127.00^{\mathrm{b}}$ & $132.03^{\mathrm{a}}$ & $10.27^{\mathrm{a}}$ & $4.96^{\circ}$ & $6.23^{d}$ & $7.81^{\circ}$ & $7.06^{c}$ & $8.28^{b}$ & $8.54^{\mathrm{b}}$ \\
\hline Sum n-3 FA & $48.84^{d}$ & $36.79^{f}$ & $43.27^{\mathrm{e}}$ & $43.55^{e}$ & $53.84^{c}$ & $56.22^{b}$ & $57.22^{\mathrm{a}}$ & $8.09_{b}$ & $4.71^{d}$ & $7.10^{\mathrm{c}}$ & $8.10^{\mathrm{b}}$ & $7.06^{c}$ & $9.06^{a}$ & $9.80^{\mathrm{a}}$ \\
\hline Sum n-6 FA & $31.85^{\mathrm{d}}$ & $43.08^{b}$ & $45.96^{\mathrm{a}}$ & $46.21^{a}$ & $31.21^{d}$ & $39.34^{\mathrm{a}}$ & $46.37^{a}$ & $3.69^{\mathrm{a}}$ & $2.69^{b}$ & $3.36^{\mathrm{a}}$ & $3.65^{\mathrm{a}}$ & $2.95^{\mathrm{ab}}$ & $3.28^{\mathrm{a}}$ & $3.40^{\mathrm{a}}$ \\
\hline Sum n-3 HUFA & $45.86^{\mathrm{C}}$ & $33.10^{f}$ & $39.75^{\ominus}$ & $40.92^{d}$ & $50.74^{\mathrm{a}}$ & $53.29^{\mathrm{b}}$ & $53.80^{a}$ & $7.78^{\mathrm{c}}$ & $4.59^{\circ}$ & $6.87^{d}$ & $7.85^{\circ}$ & $6.83^{\mathrm{d}}$ & $8.74^{a}$ & $9.44^{a}$ \\
\hline Ratio n-3/n-6 FA & $1.53^{b}$ & $0.85^{d}$ & $0.94^{d}$ & $0.95^{d}$ & $1.72^{\mathrm{a}}$ & $1.43^{\mathrm{b}}$ & $1.23^{\mathrm{c}}$ & $2.19^{d}$ & $1.75^{\mathrm{e}}$ & $2.11^{d}$ & $2.22^{d}$ & $2.39^{c}$ & $2.66^{b}$ & $2.99^{\mathrm{a}}$ \\
\hline
\end{tabular}

Means in the same row with the same superscripts under hepatopancreas or muscle are not significantly different at $P<0.05$.

FAME, fatty acid methyl esters; HUFA, highly unsaturated fatty acids.

for metabolic activity during embryonic development until the first-feeding larvae. Phospholipids are required for reproduction of $P$. vannamei (Cahu et al. 1994) and in ovarian maturation of $P$. japonicus (Alava, Kanazawa, Teshima \& Koshio 1993) as well as for growth of crustaceans (Conklin, D'Abramo, Bordner \& Baum 1980; D'Abramo, Bordner, Baum \& Conklin 1981; Kanazawa 1981; Teshima, Kanazawa \& Kakuta 1986; Baum, Conklin \& Chang 1990).

Fatty acid composition and the HUFA concentration in zoeae of $S$. serrata can be significantly influenced by broodstock diets. The quality of penaeid eggs is usually correlated with their n-3 HUFA content (Middleditch et al. 1979, 1980; Cahu et al. 1986, 1994). In this study, n-3 HUFA in zoeae from broodstock fed NF was $2.6 \%$, from $\mathrm{AD}$ was $2.3-3.9 \%$ and from $\mathrm{AD}+\mathrm{NF}$ was $3.0-6.3 \% \mathrm{DW}$ (Table 7), higher than those of zoeae from pond-sourced broodstock (2.5\% DW) (V. R. Alava, unpubl. data). Whatever the dietary treatment, DHA level in zoeae was maintained higher than EPA, suggesting that DHA is particularly required in the embryonic development of crab as it is in shrimp (Cahu et al. 1994) fish (Watanabe, Izquierdo, Takeuchi, Satoh \& Kitajima 1989). The dietary essential fatty acid requirement for crab reproduction appeared higher than the requirement for growth of juvenile crustaceans, which is generally not more than $1.0 \%$ (Kanazawa, Teshima \& Endo 1979; Kanazawa, Teshima \& Sakamoto 1985; D'Abramo 1997).

In the present experimental conditions, NF alone or in combination with $\mathrm{AD}$ promoted higher larval 
production of S. serrata than AD alone. Lipid classes and essential fatty acid contents of newly hatched zoeae improved by feeding broodstock crabs with NF in combination with $\mathrm{AD}$ containing total lipid levels from $10 \%$ to $14 \%$. Cost wise, a $10 \%$ total lipid in $\mathrm{AD}$ together with NF appeared sufficient to provide the essential lipid nutrients to crab broodstock that improved larval production and quality.

\section{Acknowledgments}

This study was supported by the European Commission (INCO-DC) through Project (ICA4-CT-200110022) 'Culture and Management of Scylla spp.' The skilled technical assistance of the staff of Crustacean Hatchery, Centralized Analytical Laboratory and Feed Mill of SEAFDEC AQD is highly appreciated.

\section{References}

Alava V.R., Kanazawa A.,Teshima S. \& Koshio S. (1993) Effect of dietary phospholipids and $n-3$ highly unsaturated fatty acids on ovarian development of kuruma prawn. Nippon Suisan Gakkaishi 59, 345-351.

Alava V.R., Quinitio E.T., de Pedro J., Orosco Z., Priolo E.M. \& Wille M. (2007) Lipids and fatty acids in wild and pondreared mud crab Scylla serrata during ovarian maturation. Aquaculture Research 38, 1468-1477.

AOAC. (1984) Official Methods of Analysis. In: Association of Official Analytical Chemists, 14th edn, (ed. by K. Herlich), p. 108, Association of Official Analytical Chemists, Washington, DC, USA.

Baum N.A., Conklin D.E. \& Chang E.S. (1990) Effect of dietary lecithin in combination with casein or crab protein on cholesterol uptake and transport in the lobster Homarus americanus. Journal of World Aquaculture Society 21, 277287.

Bray W.A., Lawrence A.L. \& Lester L.J. (1990) Reproduction of eyestalk-ablated Penaeus stylirostris fed various levels of total dietary lipid. Journal of World Aquaculture Society 21, 41-52.

Cahu C. \& Fauvel C.Aquacop. (1986) Effect of food fatty acid composition of Penaeus vannamei broodstock on egg quality. ICES, Mariculture Committee, 1986 F/28, 10pp.

Cahu C., Guillaume J.C., Stephan G. \& Chim L. (1994) Influence of phospholipid and highly unsaturated fatty acids on spawning rate and egg and tissue composition in $P e$ naeus vannamei fed semi-purified diets. Aquaculture 126. 159-170.

Cahu C. \& Quazuguel P. (1989) Lipid metabolism of Penaeus vannamei broodstock: influence of dietary lipids. In: Aquaculture Europe '89, Special Publication No. 10 (ed. by R. Billard \& N. De Pauw), pp. 45-46. European Aquaculture Society, Bredene, Belgium.
Catacutan M.R. (2002) Growth and body composition of juvenile mud crab, Scylla serrata, fed different dietary protein and lipid levels and protein to energy ratios. Aquaculture 208, 113-123.

Conklin D.E., D'Abramo L.R., Bordner C.E. \& Baum N.A. (1980) A successful purified diet for the culture of juvenile lobsters: the effect of lecithin. Aquaculture 21, 243-249.

Cuzon G. \& Guillaume J. (1997) Energy and protein: energy ratio. In: Crustacean Nutrition Advances in World Aquaculture, Vol. 6 (ed. by L.R. D'Abramo, D.E. Conklin \& D.M. Akiyama), pp. 51-70. World Aquaculture Society, Baton Rouge, LA, USA.

D'Abramo L.R. (1997) Triacylglycerols and fatty acids. In: Crustacean Nutrition (ed. by L.R. D'Abramo, D.E. Conklin \& D.M. Akiyama), pp. 71-84. World Aquaculture Society, Baton Rouge, LA, USA.

D'Abramo L.R., Bordner C.E., Baum N.A. \& Conklin D.E. (1981) Essentiality of dietary phosphatidylcholine for the survival of juvenile Iobster. Journal of Nutrition 111, 231-235.

Folch J., Lees M. \& Sloane-Stanley G.H. (1957) A simple method for the isolation and purification of total lipids from animal tissues. Journal of Biology and Chemistry 226, 497509.

Galgani M.L., Goguenheim J., Galgani F. \& Cuzon G. (1989) Diets in relation to reproduction of Penaeus vannamei and Penaeus stylirostris in captivity. Aquaculture 80, 97-109, (in French).

Gomez K.K. \& Gomez A.A. (1984) Statistical Procedures for Agricultural Research. The International Rice Research Institute, Los Banos, Laguna, Phillipines 294pp.

Kanazawa A. (1981) Penaeid nutrition. In: Proceedings of the Second International Conference of Aquaculture Nutrition: Biochemical and Physiological Approaches to Shellfish Nutrition (ed. by G.D. Pruder, C.J. Langdon \& D.E. Conklin), pp. 87-105. World Mariculture Society, Louisiana State University, Baton Rouge, Louisiana, USA.

Kanazawa A., Teshima S. \& Endo M. (1979) Requirements of prawn, Penaeus japonicus for essential fatty acids. Memoirs of Faculty of Fisheries Kagoshima University 28, 27-33.

Kanazawa A., Teshima S. \& Sakamoto M. (1985) Effects of dietary lipids, fatty acids, and phospholipids on growth and survival of prawn (Penaeus japonicus) larvae. Aquaculture 50, 39-49.

Lepage G. \& Roy C.C. (1984) Improved recovery of fatty acids through direct esterification without prior extraction and purification. Journal of Lipid Research 25, 1391-1396.

Middleditch B.S., Missler S.R., Ward D.G., McVey J.P., Brown A. \& Lawrence A.L. (1979) Maturation of penaeid shrimp: dietary fatty acids. Proceedings of theWorld Mariculture Society 10, 472-476.

Middleditch B.S., Missler S.R., Hines H.B., McVey J.P., Brown A., Ward D.G. \& Lawrence A.L. (1980) Metabolic profiles of penaeid shrimp: dietary lipids and ovarian maturation. Journal of Chromatography 195, 359-368. 
Millamena O.M. (1989) Effect of fatty acid composition of broodstock diet on tissue fatty acid patterns and egg fertilization and hatching in pond-reared Penaeus monodon. Asian Fisheries Science 2, 127-134.

Millamena O.M. \& Quinitio E.T. (2000) The effects of diets on reproductive performance on eyestalk ablated and intact mud crab Scylla serrata. Aquaculture 181, 81-90.

Nascimento I.A., Bray W.A., Leung Trujillo J.R. \& Lawrence A. (1991) Reproduction of ablated and unablated Penaeus schmitti in captivity using diets consisting of fresh-frozen natural and dried formulated feeds. Aquaculture 99, 387398.

Quinitio E.T. \& Parado-Estepa F.D. (2003) Biology and Hatchery of Mud Crabs Scylla spp. Aquaculture Extension Manual No. 34. Southeast Asian Fisheries Development Center,
Aquaculture Department, Tigbauan, Iloilo, Philippines, 42pp.

Sheen S.S. (2000) Dietary cholesterol requirement of juvenile mud crab Scylla serrata. Aquaculture 189, 277-285.

Sheen S. \& Wu S. (1999) The effects of dietary lipid levels on the growth response of juvenile mud crab Scylla serrata. Aquaculture 175, 143-153.

Teshima S., Kanazawa A. \& Kakuta Y. (1986) Role of dietary phospholipids in the transport of ${ }^{14} \mathrm{C}$ tripalmitin in the prawn. Bulletin of Japan Society of Scientific Fisheries $\mathbf{5 2}$. 519-524.

Watanabe T., Izquierdo M., Takeuchi T., Satoh S. \& Kitajima C. (1989) Comparison between eicosapentaenoic and docosahexaenoic acids in terms of essential fatty acid efficacy in larval red seabream. Nippon Suisan Gakkaishi 55, 16351640 . 